\title{
Direkte Messung des Stromflusses durch G-Quadruplex-DNA mithilfe von mechanisch kontrollierbaren Bruchkontaktelektroden***
}

\author{
Shou-Peng Liu, Samuel H. Weisbrod, Zhuo Tang, Andreas Marx, Elke Scheer und Artur Erbe*
}

Der Bedarf an miniaturisierten Bauteilen für zukünftige Anwendungen in der Nanoelektronik hat zur Suche nach neuen molekularen Schaltelementen geführt. DNA ist besonders interessant für nanoelektronische Stromkreisen aufgrund ihrer vorhersagbaren Ausmaße und der selbstorganisierten Stapelung der Nucleobasenpaare. ${ }^{[1.2]}$ In den letzten Jahren hat der Ladungstransport durch Doppelstrang-DNA (dsDNA) größere Beachtung gefunden, weil dsDNA-Nanodrähte potenziell als Bausteine in nanoelektronischen Stromkreisen genutzt werden können. Hierbei spielt die einzigartige Fähigkeit der DNA zur Selbstorganisation, ${ }^{[1.2]}$ die außerdem für biochemische Sensoren interessant ist, ${ }^{[3]}$ eine zentrale Rolle. Theoretische Studien deuten auf eine relativ hohe Elektronenleitfähigkeit von DNA im Fall einer ungestörten Überlappung der $\pi$-Orbitale hin. ${ }^{[2]}$ In früheren experimentellen Arbeiten berichteten einige Gruppen über DNAMoleküle mit hoher Leitfähigkeit, ${ }^{[4-6]}$ wohingegen andere Experimente vorwiegend sehr geringe Leitfähigkeiten der untersuchten DNA aufzeigten, ${ }^{[7-10]}$ wahrscheinlich aufgrund von unterschiedlicher Kontaktgestaltung ${ }^{[11.12]}$ sowie variabler Sequenz und flexibler Konformation der DNA. Kürzlich jedoch wurde berichtet, dass kurze dsDNA mit G-C-Sequenz eine höhere Leitfähigkeit aufweist als solche mit A-T-Sequenz. ${ }^{[13-15]}$ Bestimmte guaninreiche DNA-Sequenzen, wie man sie in den Telomeren an den Enden der Chromosomen findet, können sich zu stabilen Quadruplex-Strukturen falten, in denen Stapel aus mehreren G-Quartetten vorliegen. ${ }^{[16-18]}$ G-Quartette bildende Sequenzen wurden auch in den Promotoren von Protoonkogenen gehäuft identifiziert. ${ }^{[19]}$

Hier berichten wir über direkte Messungen des Stromflusses durch einen G-Quadruplex, der kovalent in einem mechanisch kontrollierbaren Bruchkontakt zwischen zwei

[*] S.-P. Liu, Prof. Dr. E. Scheer, Dr. A. Erbe ${ }^{[+]}$

Fachbereich Physik, Universität Konstanz

Universitätsstraße 10, 78464 Konstanz (Deutschland)

Fax: (+ 49) 753-188-3091

E-Mail: Artur.Erbe@uni-konstanz.de

S. H. Weisbrod, Dr. Z. Tang, Prof. Dr. A. Marx

Fachbereich Chemie, Universität Konstanz

Universitätsstraße 10, 78464 Konstanz (Deutschland)

${ }^{+}{ }^{+}$Derzeitige Adresse: Forschungszentrum Dresden-Rossendorf, Bautzner Landstraße 400, 01328 Dresden (Deutschland)

[**] Wir profitierten von Diskussionen mit J. C. Cuevas, M. Hettler, G. Cuniberti, M. Elstner und J. Hartig. Wir danken B. Bornemann, H. Li und T. Böhler für ihre Beiträge zu dieser Publikation sowie der Alfried Krupp von Bohlen und Halbach-Stiftung, der Landesstiftung Baden-Württemberg und der DFG (SFB 513 und SPP 1243) für finanzielle Unterstützung.
Goldelektroden befestigt wurde. Der G-Quadruplex weist eine relativ hohe Leitfähigkeit auf, die auch dann bestehen blieb, wenn die Entfernung zwischen den Elektroden reversibel über eine Spanne von mehreren Nanometern variiert wurde. Diese beispiellosen Eigenschaften machen G-Quadruplexe zu interessanten Kandidaten für nanoelektronische Anwendungen, wo ohne Verlust der Leitfähigkeit unterschiedliche Abstände zwischen Elektroden überbrückt werden müssen.

Neben der üblichen Doppelhelixform können bestimmte guaninreiche DNA-Sequenzen auch stabile QuadruplexStrukturen bilden, in denen mehrere G-Quartette gestapelt sind; bei diesen Quartetten handelt es sich um ebene Anordnungen von je vier Guaninbasen, die durch acht Wasserstoffbrücken zusammengehalten werden (Abbildung 1a). Stapel aus solchen G-Quartetten können durch Kationen wie $\mathrm{K}^{+}$oder $\mathrm{Na}^{+}$stabilisiert werden, die Plätze zwischen jeweils zwei Quartetten besetzen. Aus verschiedenartigen Sequenzen können intra- oder intermolekulare G-Quadruplexe oder G- a)

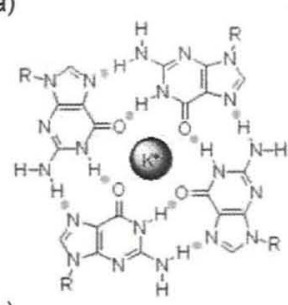

b)

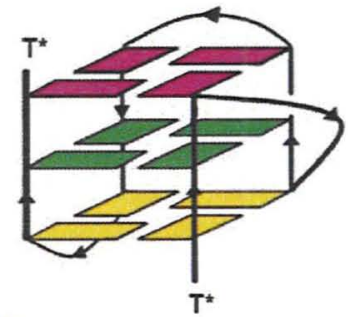

d)

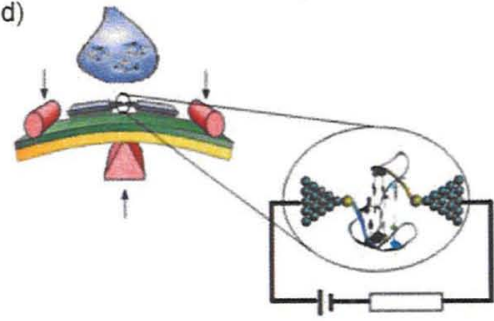

Abbildung 1. Struktur der Oligonucleotide: a) G-Quartett-Ebene mit einem zentralen Kation (graue Kugel); graue Punkte: Wasserstoffbrücken. b) G-Quadruplex-Struktur mit gemischt parallel-antiparalleler Faltung (nach einer NMR-spektroskopischen Strukturanalyse in Lösung). ${ }^{[22]}$ Schwarze Linien zeigen die Orientierung des Rückgrats. Die farbigen Rechtecke verdeutlichen die drei gestapelten G-Quartette. c) An den Molekülenden befinden sich modifizierte Thymidinbasen $\left(T^{*}\right)$, welche die Bindung zu den Goldelektroden ermöglichen. d) MKBAufbau: Eine freitragende, $100 \mathrm{~nm}$ schmale Metallbrücke wird auf einen flexiblen Träger aufgebracht. Der Träger wird gebogen, indem der zentrale Stempel gegen die äußeren Gegenlager gedrückt wird. Dabei bricht die Metallbrücke, und es entstehen zwei nah beieinander liegende Elektroden mit einzelnen Atomen an ihren Spitzen. Die DNA wird aufgebracht, indem ein Tropfen einer DNA-Lösung auf dem Probenträger platziert wird. 
Drähte (mit Längen bis in den Mikrometerbereich) gebildet werden. Für die menschliche Telomersequenz, die wir in unseren Experimenten verwendeten, wurden verschiedene Strukturen mit paralleler, ${ }^{[20]}$ antiparalleler ${ }^{[21]}$ oder gemischt parallel-antiparalleler Faltung ${ }^{[22]}$ der Stränge beschrieben.

Obwohl bisher keine Struktur im Vakuum bekannt ist, gibt es Hinweise darauf, dass G-Quadruplexe mit gestapelten G-Quartetten in der Gasphase existieren. ${ }^{[23.24]}$ Weil zwischen den Quartetten eine stärkere Überlappung der $\pi$-Elektronen auftritt, und weil lange GQuadruplex-DNA-Strukturen weniger flexibel sind als dsDNA, sollten sie eine höhere Leitfähigkeit aufweisen; diese Vermutung konnte durch Polarisierbarkeitsmessungen bestätigt werden. ${ }^{[25]}$ Darüber hinaus dürften $\mathrm{K}^{+}$- oder $\mathrm{Na}^{+}$-Ionen in den Quadruplexen die Leitfähigkeit verbessern, weil sie die Basenstapelung fördern und eine intrinsische Dotierung darstellen, ähnlich wie das Einbringen von Metallkationen ins Innere von dsDNA-Helices deren elektronische Eigenschaften verändert. ${ }^{[26-29]}$ Um den Stromfluss durch einen G-Quadruplex im Experiment zu messen, nutzten wir einen mechanisch kontrollierbaren Bruchkontakt (MKB; siehe Abbildung 1d und die Hintergrundinformationen). Dabei verbindet ein einzelnes Oligonucleotid, das einen GQuadruplex aus drei G-Quartetten bilden kann, die Elektroden (Abbildung $1 \mathrm{~b}$ und $\mathrm{c}$ ). Der G-Quadruplex wurde so modifiziert, dass das $\pi$-System der Nucleobasen am oberen und unteren Ende des Quadruplexes direkt über eine ThiolGold-Bindung mit den Goldelektroden des MKB verbunden werden kann. ${ }^{[30]}$

Zuerst vermaßen wir den Ladungstransport in Abhängigkeit vom Elektrodenabstand, wobei die Elektroden durch das Oligonucleotid G1 (5'-( $\left.\left.T^{*} \mathrm{G}_{3}[\mathrm{TTAGGG}]_{3} \mathrm{~T}^{*}\right)-3^{\prime}\right)$ verbunden wurden, das stabile G-Quadruplexe bildet. Dabei steht $T^{*}$ für die modifizierte Thymidinbase ${ }^{[30]}$ deren Schwefelatome die Bindung der Moleküle an die Metallelektroden ermöglichen (Abbildung $1 \mathrm{c}$ ). Die Abbildungen $2 \mathrm{a}-\mathrm{c}$ zeigen den gemessenen Widerstand, während der Abstand der Elektroden für zwei Proben von G1 verkürzt und verlängert wurde. In Abbildung 2a (Probe Nr. 1) weist der Widerstand beim Öffnen und Schließen des Spalts zwischen den Elektroden ein ausgeprägtes Plateau auf, das einem Abstandsbereich von etwa $2 \mathrm{~nm}$ entspricht. Der Widerstandswert im Plateaubereich schwankt und liegt in der Größenordnung um $10^{8} \Omega$. Dieses Verhalten ist in etwa 30 Zyklen reproduzierbar. Danach verschwindet das Plateau schließlich (grüne und blaue Kurve), und die veränderten $I$ - $V$-Kennlinien zeigen den
Verlust des Moleküls im Kontakt an. Ein ähnliches Verhalten wird bei der Wiederholung der Experimente mit Probe Nr. 2 beobachtet (Abbildung $2 \mathrm{~b}$ und $\mathrm{c}$ ), mit dem kleinen Unterschied, dass die Plateaus beim Öffnen des Spalts zwischen den Elektroden ausgeprägter auftreten. Wiederum bleiben die Kurven mehrere Zyklen lang stabil. Die Breite des Plateaus schwankt zwischen 0.5 und $3.5 \mathrm{~nm}$. Anders als in gleichartigen Untersuchungen mit dsDNA, bei denen ebenfalls Plateaus beim Verändern des Elektrodenabstands gefunden wurden ${ }^{[31]}$ ist der stufenförmige Verlauf in vielen Details reproduzierbar; die Stufen treten bei denselben Abständen und denselben Widerstandswerten auf. Für andere Molekül- und Atomkontakte unterscheiden sich die Kurven von Öffnung zu Öffnung, und charakteristische Widerstands- oder Abstandswerte können nur statistisch ermittelt werden. ${ }^{[15.32]}$ Reproduzierbare Öffnungs- und Schließzyklen werden beobachtet, wenn die Elektroden maximal $4 \mathrm{~nm}$ voneinander entfernt werden (bis zu einem Widerstand oberhalb $10^{10} \Omega$ ). Wird der Abstand weiter vergrößert, so verändert sich der stufenförmige Verlauf, oder er verschwindet ganz. Bei einem solchen vollständigen Bruch werden Gold-Gold-Bindungen gebrochen, ${ }^{[33]}$ und die lokale Geometrie wird komplett verändert.

Wir deuten die Plateaus als charakteristisch für das Entfalten und Falten der Moleküle im Kontakt. In diesen Kontakten wirkt eine streckende Kraft senkrecht zu den GQuartett-Ebenen, die dadurch gegeneinander verschoben werden, was zu den Fluktuationen des Widerstandswerts in 
der Plateauphase führt. Jüngste Messungen zur Bindungsstärke innerhalb von G-Quadruplex-Strukturen ergaben Kräfte zwischen 25 und $50 \mathrm{pN}{ }^{[34.35]}$ was mehrere Zehnerpotenzen unter den veranschlagten Werten für typische ThiolGold-Bindungen $(1.5 \mathrm{nN})$ und Bindungen im Metall $(1 \mathrm{nN})$ liegt. ${ }^{[35]}$ Der Widerstand bleibt in der Plateauphase nahezu konstant, solange die G-Quadruplex-Konformation noch vorliegt. Das leicht abweichende Verhalten von Probe Nr. 1 und Nr. 2 kann unterschiedlichen Bindestellen der G-Quadruplexe auf den Elektroden zugeschrieben werden.

Die Interpretation der Öffnungs- und Schließkurven wird unterstützt durch die Strom-Spannungs-Kennlinien, die bei verschiedenen Streckungszuständen gemessen wurden (Abbildung 3). Als Anhaltspunkt für den Molekülzustand während des Streckens maßen wir den Widerstand $R$ während der Öffnungs- und Schließkurven bei einer Spannung von $V_{\mathrm{sd}}=$ $100 \mathrm{mV}$. Abbildung 3d gibt die drei Regionen an, bei denen die $I$ - $V$-Kurven in Abbildung $3 \mathrm{a}-\mathrm{c}$ bestimmt wurden. Für kleine Widerstände $R<10^{6} \Omega$ sind die $I$ - $V$-Kurven in einem Spannungsbereich bis ungefähr $1 \mathrm{~V}$ weitgehend linear, was wir dem Tunneln von Elektronen durch eine Barriere zuordnen. ${ }^{[36]}$ Es gibt keine Hinweise auf einen Elektronentransport durch die Molekülorbitale, weil der Stromfluss durch direktes Tunneln den Strom, der durch das Molekül fließen kann, um eine Zehnerpotenz übersteigt. In der Plateauregion, für $10^{7} \Omega<R(V=100 \mathrm{mV})<10^{9} \Omega$, weisen die $I$ $V$-Kurven eine typische S-Form auf, wie es bereits früher für andere DNA-Derivate gefunden wurde. ${ }^{[1.2]}$ Jedoch ist der Strompegel unserer Proben - mehrere hundert Nanoampere bei einem Widerstand von ungefähr $0.7 \mathrm{~V}$ - größer als die Werte, die in den meisten Fällen für dsDNA berichtet wurden. ${ }^{[1]}$ Wir beschränkten die Stromstärke in unserem Aufbau auf $1 \mu \mathrm{A}$, da beim Überschreiten dieses Werts gelegentlich der Kontakt zerstört wurde. Wir merken an, dass normale Tunnel- oder Halbleitertransportmodelle die $I$ - $V$ Kurven nicht beschreiben können. Wir finden gewöhnlich eine Unterdrückung des Stromflusses bei einer Spannung von $0 \mathrm{~V}$, was auftreten kann, wenn die Ladeenergie der Elektronen auf den Molekülen größer ist als die Kopplung zwischen den Elektroden und den Molekülen. ${ }^{[37]}$ Die relativen Größen dieser Energien hängen von der Verbindung der Moleküle zum Metall und der Geometrie der Kontakte ab. Bei unseren Miessungen hängt die Größe des Spannungsbereichs mit reduzierter Leitfähigkeit von dem Ausmaß der Streckung und vom Widerstand ab. Aufgrund der ausgeprägten Widerstandsschwankungen ist eine genaue Korrelation kaum aufzustellen, aber tendenziell führen größere Abstände zu höheren Spannungen. In mehreren Fällen fanden wir ein Hystereseverhalten mit systematisch höherem Stromfluss, wenn der absolute Spannungswert verringert wurde. Diese Asymmetrien und Hysteresen erschweren eine quantitative Beschreibung der Kurvenform. Die Polarität der Asymmetrien wechselt jedoch von Kontakt zu Kontakt, was darauf hinweist, dass die Nichtlinearität tatsächlich aufgrund des Stromtransports durch ein einzelnes (oder einige wenige) Moleküle entsteht. ${ }^{[38]}$

Wenn der Kontakt über die Plateauregion hinaus geöffnet wird, wechseln die $I$ - $V$-Kurven wieder zum anfänglichen, nahezu linearen Verhalten, was wir wiederum als Tunneln deuten. In der überstreckten Stellung ist die Konformation des Moleküls ein DNA-Einzelstrang. Ladungstransport durch Tunneln ist der typische Transportmechanismus durch Einzelstrang-DNA. ${ }^{[1,15]}$

Zum Abschluss untersuchten wir auch die Transporteigenschaften des Kontroll-Oligonucleotids $\mathrm{C} 1 \quad\left(5^{\prime}-\mathrm{d}\left(\mathrm{T}^{*} \mathrm{C}_{3}\right.\right.$ [TTACCC $\left.\left.]_{3} \mathrm{~T}^{*}\right)-3^{\prime}\right)$, das keine Quadruplex-Struktur einnimmt ${ }^{[39]}$ und nur im sauren Bereich $(\mathrm{pH}<5.9)$ ein I-Motiv bilden könnte ${ }^{\left[{ }^{[0]}\right]}$ Es wurden keine reproduzierbaren Plateaus
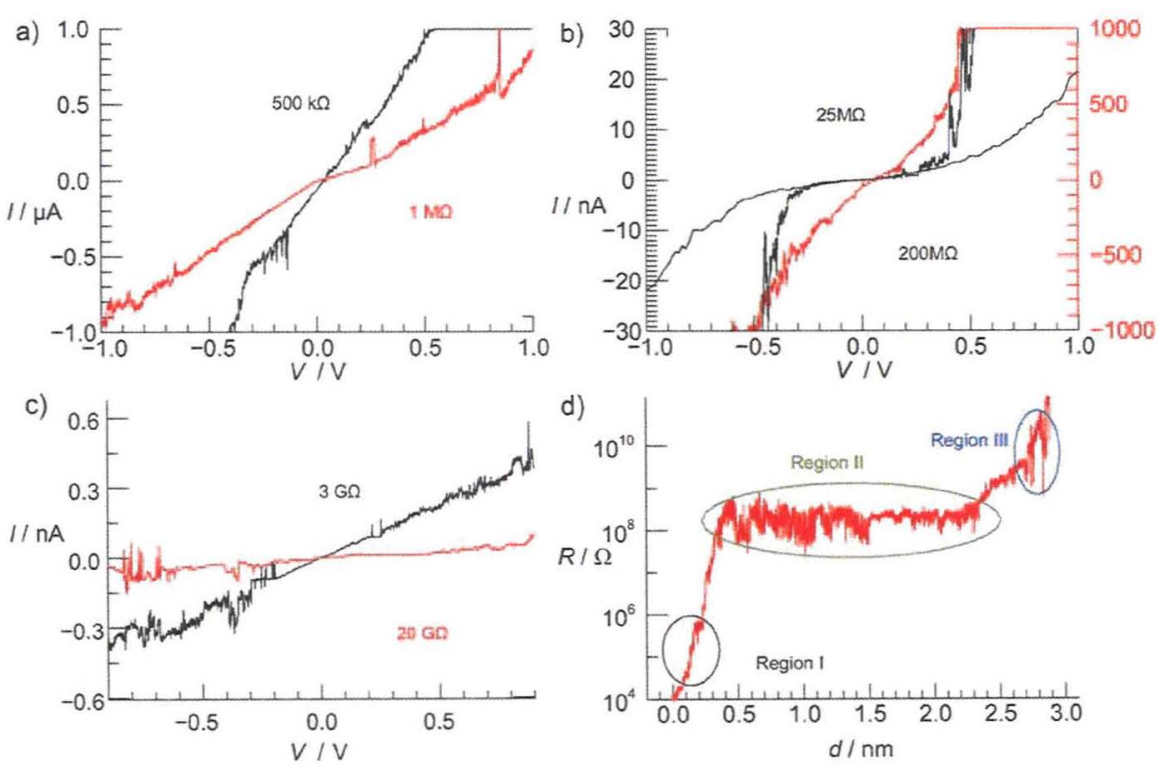

d)

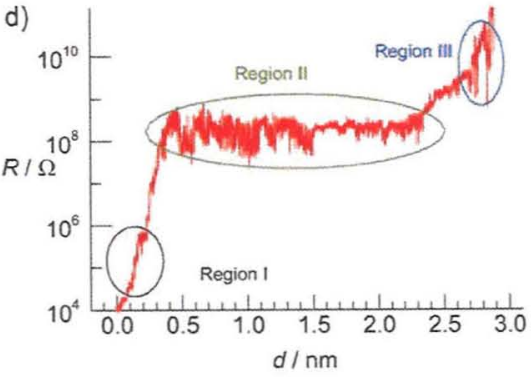

Abbildung 3. I-V-Kennlinien für Probe Nr. 1 und Nr. 2 (a-c) bei verschiedenen Stufen des Entfaltens (d). a) Typische Kurven in Region I, b) in Region II (Plateauphase) und c) in Region III. d) In der Auftragung des Widerstands gegen den Elektrodenabstand für Probe Nr. 1 sind die Regionen I-III definiert. in den Öffnungs- und Schließkurven beobachtet (Abbildung 2d), und die $I$ - $V$-Kurven waren entweder unstet (Sprünge beim Abtasten der Spannung) oder annähernd linear, wie man es für Tunneln durch eine Barriere erwartet. Dieses Verhalten wurde auch für Einzelstrang-DNA experimentell beobachtet, die über KohlenstoffNanoröhrchen kontaktiert war. ${ }^{[15]}$

Zusammenfassend haben wir gezeigt, dass G-Quadruplex-DNA bei mäßigen Transportspannungen im Bereich von $1 \mathrm{~V}$ einen beträchtlichen Strom transportieren kann. Während die $I-V$-Kennlinien nichtlinear sind und der Widerstand deshalb stark von der angelegten Spannung abhängt, hängt der Widerstand kaum vom Streckungszustand des Moleküls ab. Diese Eigenschaft ist einzigartig für molekulare Kontakte, deren Widerstand normalerweise 
bei kleinen Änderungen der Anordnung stark schwankt. Eine längenunabhängige Leitfähigkeit wurde für atomare Drähte früher bereits entdeckt, ${ }^{[32]}$ jedoch darf die Längenschwankung in diesem Fall nur wenige Ångstrom betragen. G-Quadruplexe könnten deshalb als flexible Anschlussdrähte dienen, um Mikroelektroden mit molekularen Funktionseinheiten wie molekularen Schaltern zu verbinden, die eine beträchtliche Konformationsänderung beim Schalten eingehen, z.B. photoschaltbare Azobenzolderivate. ${ }^{[30]}$

\section{Experimentelles}

$5^{\prime}-\mathrm{T}^{*}$ GGG[TTAGGG] $\mathrm{T}^{*}-3^{\prime}$ (G1) und $5^{\prime}-\mathrm{T}^{*} \mathrm{CCC}[\mathrm{TTACCC}]_{3} \mathrm{~T}^{*}-3^{\prime}$ (C1) wurden durch automatisierte DNA-Oligonucleotid-Synthese erhalten. ${ }^{[25]}$ Massenspektrometrische Analysen wiesen die Integrität der Oligonucleotide nach. Zur Immobilisierung wurden die Oligonucleotide mit PBS-Puffer auf eine Konzentration von $10 \mu \mathrm{M}$ verdünnt ( $40 \mathrm{mM} \mathrm{K}_{2} \mathrm{PO}_{4}$ und $26 \mathrm{mM} \mathrm{KH}_{2} \mathrm{PO}_{4}, \mathrm{pH}$ 6.98). In dieser Lösung (ca. $100 \mathrm{~mm} \mathrm{~K}^{+}$) sollte das 22 mere Oligonucleotid G1 eine parallele oder gemischt parallel-antiparallele Quadruplex-Struktur einnehmen (Abbildung 1), während $\mathrm{Cl}$ in der Einzelstrang-Konformation verbleibt, wie mit CD-Spektroskopie gezeigt wurde. ${ }^{[36.39]}$

Eingegangen am 4. Januar 2010

Online veröffentlicht am 26. März 2010

chwörter: Bruchkontakte - DNA - Molekulare Elektronik . Nanoelektronik

[1] D. Porath, G. Cuniberti, R. Di Felice, Top. Curr. Chem. 2004, $237,183$.

[2] R. G. Endres, D. L. Cox, R. R. P. Singh, Rev. Mod. Phys. 2004, $76,195$.

[3] K. J. Odenthal, J. J. Gooding, Analyst 2007, 132, 603.

[4] A. Y. Kasumov, M. Kociak, S. Gueron, B. Reulet, V. T. Volkov, D. V. Klinov, H. Bouchiat, Science 2001, 291, 280.

[5] A. Y. Kasumov, D. V. Klinov, P. E. Roche, S. Gueron, H. Bouchiat, Appl. Phys. Lett. 2004, 84, 1007.

[6] H.-W. Fink, C. Schönenberger, Nature 1999, 398, 407.

[7] Y. Zhang, R. H. Austin, J. Kraeft, E. C. Cox, N. P. Ong, Phys. Rev. Lett. 2002, 89, 198102.

[8] H. Cohen, C. Nogues, R. Naaman, D. Porath, Proc. Natl. Acad. Sci. USA 2005, 102, 11589.

[9] E. Braun, Y. Eichen, U. Sivan, G. Ben-Yoseph, Nature 1998, 391, 775 .

[10] D. Porath, A. Bezryadin, S. de Vries, C. Dekker, Nature 2000, 403,635 .

[11] H. Watanabe, C. Manabe, T. Shigematsu, K. Shimotani, M. Shimizu, Appl. Phys. Lett. 2001, 79, 2462.

[12] X. Guo, A. A. Gorodetsky, J. Hone, J. K. Barton, C. Nuckolls, Nat. Nanotechnol. 2008, 3, 163 .

[13] K. H. Yoo, D. H. Ha, J. O. Lee, J. W. Park, J. Kim, J. J. Kim, H. Y. Lee, T. Kawai, H. Y. Choi, Phys. Rev. Lett. 2001, 86, 8719.
[14] B. Q. Xu, P. M. Zhang, X. L. Li, N. J. Tao, Nano Lett. 2004, 4, 1105 .

[15] S. Roy, H. Vedala, A. D. Roy, D. H. Kim, M. Doud, K. Mathee, H. K. Shin, N. Shimamoto, V. Prasad, W. Choi, Nano Lett. 2008, $8,26$.

[16] C. W. Greider, E. H. Blackburn, Cell 1985, 43, 405

[17] N. W. Kim, M. A. Piatyszek, K. R. Prowse, C. B. Harley, M. D. West, P. L. Ho, G. M. Coviello, W. E. Wright, S. L. Weinrich, J. W. Shay, Science 1994, 266, 2011.

[18] J. T. Davis, G. P. Spada, Chem. Soc. Rev. 2007, 36, 296.

[19] J. L. Huppert, S. Balasubramanian, Nucleic Acids Res. 2005, 33, 2908.

[20] G. N. Parkinson, M. P. H. Lee, S. Neidle, Nature 2002, 417, 876.

[21] Y. Wang, D. J. Patel, Structure 1993, 1, 263.

[22] J. Dai, C. Punchihewa, A. Ambrus, D. Chen, R. A. Jones, D. Yang, Nucleic Acids Res. 2007, 35, 2440.

[23] M. Rueda, F. J. Luque, M. Orozco, J. Am. Chem. Soc. 2006, 128, 3608.

[24] V. Gabelica, F. Rosu, E. De Pauw, J. Lemaire, J.-C. Gillet, J.-C. Poully, F. Lecomte, G. Gregoire, J.-P. Schermann, C. Desfrancois, J. Am. Chem. Soc. 2008, 130, 1810.

[25] H. Cohen, T. Sapir, N. Borovok, T. Molotsky, R. Di Felice, A. B. Kotlyar, D. Porath, Nano Lett. 2007, 7, 981.

[26] K. Tanaka, A. Tengeiji, T. Kato, N. Toyama, M. Shionoya, Science 2003, 299, 1212.

[27] P. Aich, S. L. Labiuk, L. W. Tari, L. J. Delbaere, W. J. Roesler, K. J. Falk, R. P. Steer, J. S. Lee, J. Mol. Biol. 1999, 294, 477.

[28] A. Rakitin, P. Aich, C. Papadopoulos, Y. Kobzar, A. S. Vedeneev, J. S. Lee, J. M. Xu, Phys. Rev. Lett. 2001, 86, 3670.

[29] K. Tanaka, Y. Yamada, M. Shionoya, J. Am. Chem. Soc. 2002, 124, 8802.

[30] B. Bornemann, S. Liu, A. Erbe, E. Scheer, A. Marx, ChemPhysChem 2008, 9, 1241.

[31] N. Kang, A. Erbe, E. Scheer, New J. Phys. 2008, 10, 023030.

[32] N. Agraït, A. L. Yeyati, J. M. van Ruitenbeek, Phys. Rep. 2003, 377,81 .

[33] J. Reichert, H. B. Weber, M. Mayor, H. von Löhneysen, Appl. Phys. Lett. 2003, 82, 4137.

[34] Z. Yu, J. D. Schonhoft, S. Dhakal, R. Bajracharya, R. Hegde, S. Basu, H. Mao, J. Am. Chem. Soc. 2009, 131, 1876. [35] S. Lynch, H. Baker, S. G. Byker, D. Zhou, K. Sinniah, Chem. Eur.
J. 2009, 15, 8113 .

[36] W. Wang, T. Lee, M. A. Reed, Phys. Rev. B 2003, 68, 035416.

[37] F. Zahid, M. Paulsson, S. Datta, Handbook of Nanoscience, Engineering and Technology, CRC, Boca Raton, 2002.

[38] J. Reichert, R. Ochs, D. Beckmann, H. B. Weber, M. Mayor, H. von Löhneysen, Phys. Rev. Lett. 2002, 88, 176804.

[39] Grundsätzlich kann die Quadruplex-Struktur durch Hinzufügen bestimmter Ionen zum Puffer geändert werden. Das verändert jedoch die Gesamtleitfähigkeit der Verbindung. Daher können Änderungen der Molekülkonformation nicht detektiert werden. Das hier beschriebene Verhalten wurde bei mehreren DNASequenzen nicht gefunden, die wir mit einem identischen Aufbau und mit mehreren Wiederholungen untersucht haben.

[40] K. Guo, V. Gokhale, L. H. Hurley, D. Sun, Nucleic Acids Res. 2008, 36, 4598 . 